\title{
Restriction Space Projection Method for Position Sensor Based Force Reflection of Multi Degrees-of-Freedom Bilateral Teleoperation Systems in Unstructured Environments
}

\author{
Keehoon Kim, Wan Kyun Chung, and M. Cenk Çavuşoğlu
}

\begin{abstract}
In bilateral teleoperation system, conventional position sensor based force reflection method, known as position error feedback, may generate inaccurate force reflection directions, when motion of a slave robot is constrained by unexpected obstacles and link collisions. Restriction Space Projection method is a novel position sensor based force reflection framework that was proposed to address this issue. It provides accurate force reflection in unstructured environments when motion of a slave robot is constrained by unexpected obstacles and link collisions, regardless of kinematic dissimilarity between the master and slave manipulators of the bilateral teleoperation system. This paper discusses the applications and limitations of the Restriction Space Projection method through examples.
\end{abstract}

\section{INTRODUCTION}

In bilateral teleoperation systems, force sensors dramatically enhance the accuracy of the force reflection compared to the position sensor based force reflection. It is, however, practically burdensome to distribute enough force sensors to detect every possible physical interaction. In addition, a bilateral teleoperation system using the force sensor based force reflection is confronted by a serious problem when the force sensor cannot detect the interaction force. For example, if a slave robot collides with unexpected obstacles which are not detected by force sensors, no force is reflected to a master device. As a human operator is able to move the master device without constraint, the system becomes unstable due to the significant position difference between the master device and the slave robot. This situation can happen frequently during the operation is unstructured, cluttered, or moving environments such as underwater manipulations, prosthetic arm manipulations, or minimally invasive surgical robot operations.

On the other hand, since position sensors are typically placed on every joint of a bilateral teleoperation system, the position sensor based force reflection method is able to enhance robustness against unexpected obstacle collisions, especially, collisions occurring not at the end-effector, but at the links of the slave manipulator. In the previous study, the conventional position sensor based force reflection method, known as position error feedback method, is shown not to be able to adequately describe the direction of the force reflection especially when the bilateral teleoperation system is multi degrees-of-freedom [1]-[3]. Therefore, enhanced

K. Kim is currently with Korea Institute of Science and Technology (KIST), Seoul, Korea, W. K. Chung is with the Pohang Univ. of Science and Technology(POSTECH), Pohang, Korea, and M. C. Çavuşoğlu is with the Case Western Reserve University, Cleveland, OH, USA. position sensor based force reflection frameworks have been proposed for multi degrees-of-freedom bilateral teleoperation systems satisfying the following conditions [1]-[3]:

- The force reflection framework should be applicable to multi degrees-of-freedom bilateral teleoperation systems for all types of kinematic dissimilarity conditions shown in Table $\mathrm{I}^{1}$.

- The force reflection framework should deal with the joint constraints as well as the end-point constraint.

- The force reflection framework should be applicable in unstructured environment without a priori knowledge of the obstacles or the environment.

- The force reflection framework should be able to use force sensor signals when obstacles are detectable by force sensor.

Table I classifies bilateral teleoperation systems according to the kinematic dissimilarity conditions. The conventional position error feedback method is applicable to kinematically similar (KS) type in which one to one joint angle control is available. For kinematically dissimilar teleoperation systems, the conventional position error feedback is only applicable if the two manipulators have equal degrees of freedom and a tasks space controller with the same gain in all directions is used [1].

Previously, Kim et al. proposed a position sensor based force reflection method, called restriction space projection (RSP) method [2], [3], which is based on the projection of the restriction space encountered by the slave manipulator in contact with a rigid obstacle in the environment to the master. However, the RSP method proposed in [2], [3] is limited to specific joint constraints and kinematic dissimilarity conditions. The method proposed in [2], [3] was not able to calculate the restriction space accurately in cases with multiple obstacle collisions. The method proposed in [2], [3] was also not able to handle redundancy at the slave side at all. Kim et al. significantly extends the RSP method to the general case under any type of constraint for all of the kinematic dissimilarity conditions listed in Table I [1].

\footnotetext{
${ }^{1}$ Jacobian of the master device, $\boldsymbol{J}_{m}: \dot{\boldsymbol{q}}_{m} \in \mathbb{R}^{m} \rightarrow \dot{\boldsymbol{x}}_{m} \in \mathbb{R}^{r}$, and Jacobian of the slave manipulator, $\boldsymbol{J}_{s}: \dot{\boldsymbol{q}}_{s} \in \mathbb{R}^{n} \rightarrow \dot{\boldsymbol{x}}_{s} \in \mathbb{R}^{r}$, where $\boldsymbol{q}_{m}$ and $\boldsymbol{q}_{s}$ are the joint angles of the master and slave robot in the joint spaces, $\mathbb{R}^{m}$ and $\mathbb{R}^{n}$, respectively. $\boldsymbol{x}_{m}$ and $\boldsymbol{x}_{s}$ are the pose of the master and slave robot in the task space $\mathbb{R}^{r} . \alpha$ is an arbitrary non-zero scalar. Note that, as defined, kinematic dissimilarity is different from geometric dissimilarity. For example, if the length scale ratio of a master device is the same with that of a slave robot, it is geometrically similar but not kinematically similar.
} 
TABLE I

APPLICATION RANGE

\begin{tabular}{ccccc}
\hline Type & Description & $\begin{array}{c}\text { Conventional } \\
\text { position error feedback }\end{array}$ & $\begin{array}{c}\text { Restriction Space } \\
\text { Projection (RSP) } \\
\text { method in [2], [3] }\end{array}$ & $\begin{array}{c}\text { Restriction Space } \\
\text { Projection (RSP) } \\
\text { method proposed in [1] }\end{array}$ \\
\hline KS & $\begin{array}{c}\boldsymbol{J}_{m}=\alpha \boldsymbol{J}_{s} \\
\text { Kinematically similar haptic interface }\end{array}$ & Applicable & Applicable \\
\hline KDS-EQDOF & $\begin{array}{c}\boldsymbol{J}_{m} \neq \alpha \boldsymbol{J}_{s}, m=n \\
\text { Kinematically dissimilar haptic interface } \\
\text { with the same degrees-of-freedom }\end{array}$ & $\begin{array}{c}\text { Partially applicable with } \\
\text { single gain task space } \\
\text { controller at the slave }\end{array}$ & $\begin{array}{c}\text { Partially applicable to } \\
\text { single link collision only }\end{array}$ \\
\hline KDS-RMASTER & $\begin{array}{c}\boldsymbol{J}_{m} \neq \alpha \boldsymbol{J}_{s}, m>n \\
\text { Kinematically dissimilar haptic interface with } \\
\text { insufficient degrees-of-freedom at the slave side }\end{array}$ & Not applicable & $\begin{array}{c}\text { Partially applicable to } \\
\text { single link collision only }\end{array}$ \\
\hline KDS-RSLAVE & $\begin{array}{c}\boldsymbol{J}_{m} \neq \alpha \boldsymbol{J}_{s}, m<n \text { Applicable } \\
\text { Kinematically dissimilar haptic interface } \\
\text { with redundancy at the slave side }\end{array}$ & Not applicable & $\begin{array}{c}\text { Not applicable [3] } \\
\text { single link collision only [2] }\end{array}$ \\
\hline
\end{tabular}

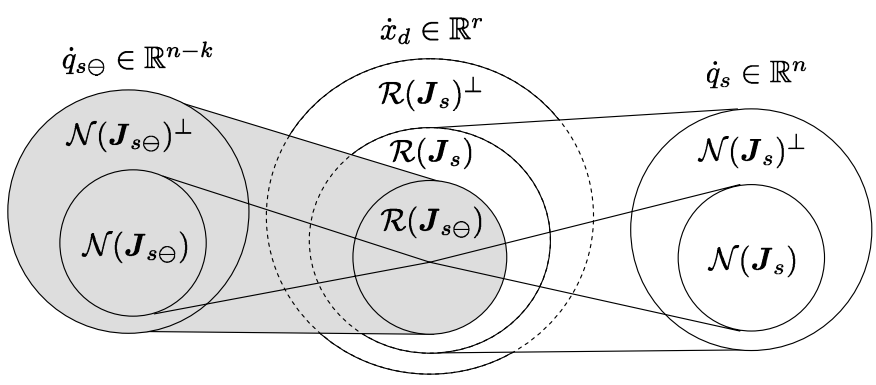

Fig. 1. Linear mapping of Instantaneous Motion Space $\left(\mathrm{IMS}=\mathcal{R}\left(J_{s \ominus}\right)\right)$ and Instantaneous Restriction Space $\left(\operatorname{IRS}_{\mathrm{G}}=\mathcal{R}\left(J_{s}\right)^{\perp}\right.$ and $\operatorname{IRS}_{\mathrm{E}}=$ $\left.\mathcal{R}\left(\boldsymbol{J}_{s}\right) \cap \mathcal{R}\left(\boldsymbol{J}_{s \ominus}\right)^{\perp}\right)$.

Section II summarizes the concept of the Instantaneous Restriction Space (IRS) and the calculation and the implementation of the IRS in the RSP framework. Section III shows how the RSP method is applied to kinematically dissimilar bilateral teleoperation systems through examples followed by discussion of applications and limitation of the RSP method in Section IV.

\section{Restriction Space Projection Method}

The concepts of Instantaneous Motion Space (IMS) and Instantaneous Restriction Space (IRS) are introduced in [1][3] as follows:

IMS : Convex cone of all possible velocity vector that the manipulator can kinematically achieve ${ }^{2}$.

IRS : Algebraic complement of instantaneous motion space (IMS).

IRS $_{\mathrm{G}}$ : IRS caused by insufficient degrees-of-freedom.

IRS $_{\mathrm{E}}$ : IRS caused by exogenous constraints.

IMS and IRS can be defined mathematically as follows when joints are bidirectionally constrained:

$$
\begin{aligned}
\mathrm{IMS} & =\mathcal{R}\left(\boldsymbol{J}_{s \ominus}\right) \\
\mathrm{IRS}_{\mathrm{G}} & =\mathcal{R}\left(\boldsymbol{J}_{s}\right)^{\perp} \\
\mathrm{IRS}_{\mathrm{E}} & =\mathcal{R}\left(\boldsymbol{J}_{s}\right) \cap \mathcal{R}\left(\boldsymbol{J}_{s \ominus}\right)^{\perp}
\end{aligned}
$$

\footnotetext{
${ }^{2}$ When there are no half space type constraints (resulting from unidirectional constraints), IMS becomes a subspace, and IRS becomes the orthogonal complement subspace of IMS
}

$\mathcal{R}(\cdot)$ stands for the range space of $(\cdot) .(\cdot)^{\perp}$ is the orthogonal complementary space of $(\cdot) . \boldsymbol{J}_{s}=\partial \boldsymbol{x}_{s} / \partial \boldsymbol{q}_{s} \in \mathbb{R}^{r \times n}$ where $\boldsymbol{x}_{s} \in \mathbb{R}^{r}$ and $\boldsymbol{q}_{s} \in \mathbb{R}^{n}$ are the pose and the joint angles of the slave robot. $\boldsymbol{J}_{s \ominus} \in \mathbb{R}^{r \times n}$ is defined as an inherited Jacobian from $\boldsymbol{J}_{s}$ and the columns of $\boldsymbol{J}_{s \ominus}$ corresponding to the constrained joints are replaced by zero column vectors. Similarly, $\dot{\boldsymbol{q}}_{s \ominus}$ is an inherited joint vector from $\dot{\boldsymbol{q}}_{s}$ and the elements of $\dot{\boldsymbol{q}}_{s \ominus}$ correspoinding to the constrained joints are replaced by zeros. In order to detect the constrained joints, we introduce a certain threshold, $\varepsilon$, to determine whether the cause of the joint angle errors, $\boldsymbol{e}_{s q}=$ $\left[e_{s q 1}, e_{s q 2}, \cdots, e_{s q i}, \cdots, e_{s q n}\right]^{T}$, come from the exogenous motion constraint (i.e, IRS $\mathrm{E}$ ) or the free motion control errors. In other words, when the joint angle error is larger than the $\varepsilon$, the corresponding column of $\boldsymbol{J}_{s \ominus}$ is replaced by a zero vector. Mathematically, $\boldsymbol{J}_{s \ominus}$ and $\dot{\boldsymbol{q}}_{s \ominus}$ are defined as follows:

$$
\begin{aligned}
\boldsymbol{J}_{s \ominus} & =\boldsymbol{J}_{s} \boldsymbol{D} \\
\boldsymbol{q}_{s \ominus} & =\boldsymbol{q}_{s} \boldsymbol{D}
\end{aligned}
$$

where

$$
\boldsymbol{D}=\operatorname{diag}\left[d_{1}, d_{2}, \cdots, d_{i}, \cdots, d_{n}\right]
$$

and

$$
d_{i}=\left\{\begin{array}{ll}
0, & \text { if } e_{s q i}>\epsilon \\
1, & \text { if } e_{s q i}<\epsilon
\end{array}\right\}
$$

The threshold should be larger than the expected free motion control errors. It determines the sensitivity to trigger the proposed method. $\mathcal{R}\left(\boldsymbol{J}_{s}\right)^{\perp}$ is the restriction space that the slave robot cannot reach due to the insufficient degreesof-freedom, i.e, $\mathrm{IRS}_{\mathrm{G}}$, even if there is no exogenous motion constraint. If the motion of the slave robot is constrained, IMS becomes $\mathcal{R}\left(\boldsymbol{J}_{s \ominus}\right)$. IRS $\mathrm{IE}_{\mathrm{E}}$ becomes $\mathcal{R}\left(\boldsymbol{J}_{s}\right) \cap \mathcal{R}\left(\boldsymbol{J}_{s \ominus}\right)^{\perp}$. Fig. 1 shows the linear mapping of IMS, $\operatorname{IRS}_{\mathrm{E}}$ and $\operatorname{IRS}_{\mathrm{G}}$.

\section{A. Calculation of IRS}

In order to calculate $\mathrm{IRS}_{\mathrm{G}}$ and IRS $\mathrm{S}_{\mathrm{E}}$, two RSP matrices are defined, $\boldsymbol{R}_{G}$ and $\boldsymbol{R}_{E}$.

From the definition of IRS $\mathrm{G}_{\mathrm{G}}$ in Eq.(2), $\boldsymbol{R}_{G}$, which maps the task space pose error into the reflection force in $\operatorname{IRS}_{\mathrm{G}}$, 
can be defined as:

$$
\begin{aligned}
& \boldsymbol{R}_{G}: \quad \boldsymbol{e}_{s x} \in \mathbb{R}^{r} \rightarrow \boldsymbol{F}_{R G} \in \mathrm{IRS}_{\mathrm{G}}, \\
& \boldsymbol{R}_{G}=-K_{G}\left(\boldsymbol{I}-\boldsymbol{J}_{s} \boldsymbol{J}_{s}^{\#}\right),
\end{aligned}
$$

where $(\cdot)^{\#}$ is the pseudo-inverse of $(\cdot) . \boldsymbol{e}_{s x}\left(=\boldsymbol{x}_{d}-\boldsymbol{x}_{s}\right)$ is the task space pose error. $r$ is the workspace dimension. $K_{G}$ is a scalar force gain to adjust the magnitude of the reflection force, $\boldsymbol{F}_{R G}$.

Similarly, from the definition of IRS $_{\mathrm{E}}$ in Eq.(3), $\boldsymbol{R}_{E}$, which maps the task space pose error into the reflection force in $\operatorname{IRS}_{\mathrm{E}}$, can be defined as:

$$
\begin{aligned}
& \boldsymbol{R}_{E}: \quad \boldsymbol{e}_{s x} \in \mathbb{R}^{r} \rightarrow \boldsymbol{F}_{R E} \in \mathrm{IRS}_{\mathrm{E}} \\
& \boldsymbol{R}_{E}=-K_{E}\left(\boldsymbol{I}-\boldsymbol{J}_{s \ominus} \boldsymbol{J}_{s \ominus}^{\#}\right) \boldsymbol{J}_{s} \boldsymbol{J}_{s}^{\#}
\end{aligned}
$$

where $K_{E}$ is a scalar force gain.

Therefore, the total reflection force, $\boldsymbol{F}_{R}$ can be calculated as:

$$
\begin{aligned}
\boldsymbol{F}_{R}= & \boldsymbol{F}_{R G}+\boldsymbol{F}_{R E}=\boldsymbol{R}_{G} \boldsymbol{e}_{s x}+\boldsymbol{R}_{E} \boldsymbol{e}_{s x} \\
= & -\left\{K_{G}\left(\boldsymbol{I}-\boldsymbol{J}_{s} \boldsymbol{J}_{s}^{\#}\right)\right. \\
& \left.+K_{E}\left(\boldsymbol{I}-\boldsymbol{J}_{s \ominus} \boldsymbol{J}_{s \ominus}^{\#}\right) \boldsymbol{J}_{s} \boldsymbol{J}_{s}^{\#}\right\} \boldsymbol{e}_{s x} .
\end{aligned}
$$

Though the proposed RSP method calculates the direction of the restriction space, it cannot estimate the magnitude. Thus, users should scale $K_{E}$ and $K_{G}$ to fit for their applications considering weighting between $\boldsymbol{F}_{R G}$ and $\boldsymbol{F}_{R E}$. Note that we can adjust the force gains, $K_{G}$ and $K_{E}$, independently since $\boldsymbol{F}_{R G} \in \mathrm{IRS}_{\mathrm{G}}$ and $\boldsymbol{F}_{R E} \in \mathrm{IRS}_{\mathrm{E}}$ are in orthogonal complementary space.

\section{B. Implementation of IRS using RSP method}

A general form of the proposed RSP method can be implemented as shown in Fig.2. When the desired command, $\dot{\boldsymbol{x}}_{d}$, is transferred from the master side, it is projected to the range space of the slave robot, $\mathcal{R}\left(\boldsymbol{I}-\boldsymbol{R}_{G}\right)$. Then, the desired joint velocities are calculated from the projected desired command, $\dot{\boldsymbol{x}}_{p}$ and the desired joint angle of the slave robot, $\boldsymbol{q}_{s d}$, through inverse kinematics (IK). The local controller of the slave $\operatorname{robot}^{3}, \boldsymbol{K}_{s}$, moves the slave robot to follow the desired pose. At the same time, the external torques are distributed to joints due to the obstacle collisions, $\boldsymbol{\tau}_{\text {ext }} . \boldsymbol{P}_{s}$ stands for the slave robot dynamics. The pose error, $\boldsymbol{e}_{s x}$, is calculated from $\boldsymbol{x}_{d}$ and $\boldsymbol{x}_{s} . \boldsymbol{q}_{s}$ is joint angle. FK is a forward kinematic mapping from $\boldsymbol{q}_{s}$ to $\boldsymbol{x}_{s}$. From Eq.(9), $\boldsymbol{R}_{G}$ can be calculated from the Jacobian, $\boldsymbol{J}_{s}$. Then, $\boldsymbol{F}_{R G}=\boldsymbol{R}_{G} \cdot \boldsymbol{e}_{s x}$. From $\boldsymbol{J}_{s}$ and $\boldsymbol{e}_{s q}, \boldsymbol{J}_{s \ominus}$ can be calculated as explained in Section III and $\boldsymbol{F}_{R E}=\boldsymbol{R}_{E} \cdot \boldsymbol{e}_{s x}$ from Eq.(11). $\boldsymbol{F}_{E F}$ is the force signal detected by force sensors.

Since IRS is calculated from the joint angle error configuration in the RSP framework, an appropriate inverse kinematics solution should be sought to decrease the joint angle error avoiding the motion of the constrained joint. Thus, it is possible to allow the human operator moves the master device in larger IMS. The following inverse

\footnotetext{
${ }^{3}$ The proposed RSP works regardless of the local control algorithm of the slave robot.
}

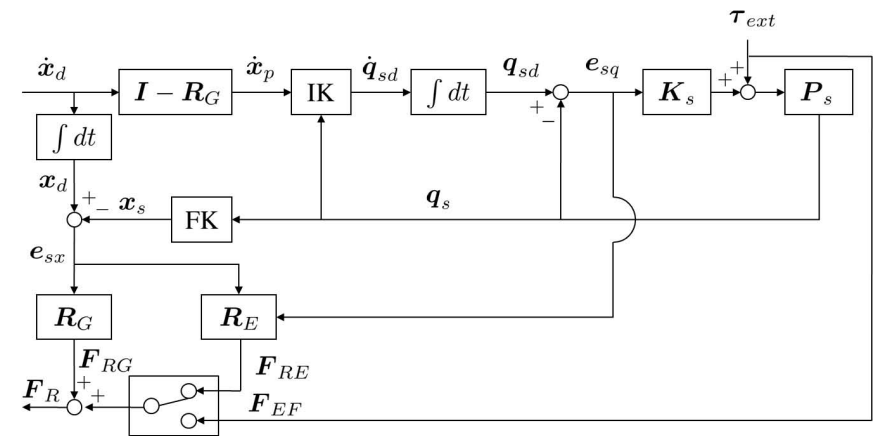

Fig. 2. Implementation of Restriction Space Projection method in a teleoperation system. $\dot{\boldsymbol{x}}_{d}$ is the desired command transferred from the master device. $\dot{\boldsymbol{x}}_{p}$ is the projected desired command onto the range space of the slave robot. IK and FK stand for inverse and forward kinematic maps. $\boldsymbol{q}_{s d}$ and $\boldsymbol{q}_{s}$ are the desired angle and the actual angle of the slave robot. $\boldsymbol{e}_{s q}=\boldsymbol{q}_{s d}-\boldsymbol{q}_{s}$. The local controller and the dynamics of the slave robot. $\boldsymbol{F}_{\text {ext }}$ is the external forces caused by obstacles. $\boldsymbol{e}_{s x}=\boldsymbol{x}_{d}-\boldsymbol{x}_{s}$ where, $\boldsymbol{x}_{d}$ and $\boldsymbol{x}_{s}$ are the desire pose and the actual pose of the slave robot. $\boldsymbol{R}_{G}$ and $\boldsymbol{R}_{E}$ are the RSP matrices. $\boldsymbol{F}_{R}, \boldsymbol{F}_{R G}$, and $\boldsymbol{F}_{R E}$ are the total reflection force, the reflection force by $\mathrm{IRS}_{\mathrm{G}}$, and the reflection force by IRS $_{\mathrm{E}}$ respectively.

kinematics solution inspired by the inverse kinematics using a potential function as a second manipulation variable [4], [5] is proposed in [1].

$$
\begin{aligned}
\dot{\boldsymbol{q}}_{s d} & =\boldsymbol{J}_{s}^{\#} \dot{\boldsymbol{x}}_{d} \\
& +\left(\boldsymbol{I}_{n}-\boldsymbol{J}_{s}^{\#} \boldsymbol{J}\right)\left(-k_{1}{\frac{\partial p}{\partial \boldsymbol{q}_{s d}}}^{T}-k_{2} \frac{\partial p}{\partial \boldsymbol{q}_{s d}}{ }^{T} \frac{\partial p}{\partial \boldsymbol{q}_{s d}} \boldsymbol{J}_{s}^{\#} \dot{\boldsymbol{x}}_{d}\right),
\end{aligned}
$$

where $p=\frac{1}{2} \boldsymbol{e}_{s q}^{T} \boldsymbol{e}_{s q}=\frac{1}{2}\left(\boldsymbol{q}_{s d}-\boldsymbol{q}_{s}\right)^{T}\left(\boldsymbol{q}_{s d}-\boldsymbol{q}_{s}\right), k_{1}>0$, and $k_{2}=\left\|\frac{\partial p}{\partial \boldsymbol{q}_{s d}}\left(\boldsymbol{I}_{n}-\boldsymbol{J}_{s}^{\#} \boldsymbol{J}_{s}\right)\right\|^{-2}$. Unless $\frac{\partial p}{\partial \boldsymbol{q}_{s d}}\left(\boldsymbol{I}_{n}-\boldsymbol{J}_{s}^{\#} \boldsymbol{J}_{s}\right)=$ $\mathbf{0}, \dot{\boldsymbol{q}}_{s d}$ from Eq.(13) contributes to decrease $p$ using its null motion. The convergence of $e_{s q}$ is proved in [1].

The inverse kinematics solution in Eq.(13) changes the desired joint angle configuration to decrease the joint angle error avoiding the joint motion constraints.

\section{EXAMPLES}

This section shows how to apply the proposed RSP framework to various bilateral teleoperation systems through three examples.

\section{A. Example 1: Two degrees-of-freedom manipulator in three dimensional cartesian task space}

Consider a two degrees-of-freedom planar slave robot in three dimensional cartesian space as shown in Fig. 3 (KDSRMASTER in Table I). The joint configuration and link lengths are assumed as: $q_{s 1}=0, q_{s 2}=\pi / 4, l_{1}=0.2$, and $l_{2}=0.3$. The pose error is assumed to be $e_{s x}=$ $\left[e_{s x}, e_{s y}, e_{s z}\right]^{T}$ : The Jacobian of the manipulator is:

$$
\boldsymbol{J}_{s}=\left[\begin{array}{ll}
\boldsymbol{J}_{s 1} & \boldsymbol{J}_{s 2}
\end{array}\right]=\left[\begin{array}{cc}
-0.3 / \sqrt{2} & -0.3 / \sqrt{2} \\
0.2+0.3 / \sqrt{2} & 0.3 / \sqrt{2} \\
0 & 0
\end{array}\right] .
$$


From Eq.(9),

$$
\begin{aligned}
\boldsymbol{R}_{G} & =-K_{G}\left(\boldsymbol{I}-\boldsymbol{J}_{s} \boldsymbol{J}_{s}^{\#}\right)=K_{G}\left[\begin{array}{ccc}
0 & 0 & 0 \\
0 & 0 & 0 \\
0 & 0 & 1
\end{array}\right], \\
\boldsymbol{F}_{R G} & =\boldsymbol{R}_{G} \boldsymbol{e}_{s x}=-K_{G}\left[\begin{array}{lll}
0 & 0 & e_{s z}
\end{array}\right]^{T} .
\end{aligned}
$$

From Eq.(15) and Eq.(16), the RSP method gives that the $z$-direction is the restriction space when $e_{s z}$ is non zero.

We will calculate two different IRS $_{\mathrm{E}}$ for two constraint conditions: $i)$ the first joint is constrained $\left(e_{s q 1}>\varepsilon\right.$ and $\left.\left.e_{s q 2}<\varepsilon\right) ; i i\right)$ the second joint is constrained $\left(e_{s q 1}<\varepsilon\right.$ and $\left.e_{s q 2}>\varepsilon\right)^{4}$, when the joint angle error, $e_{s q}=\left[e_{s q 1}, e_{s q 2}\right]^{T}$.

When the first joint is constrained (or when the first joint angle error is significant, i.e., $e_{s q 1}>\varepsilon$ ),

$$
\boldsymbol{J}_{s \ominus 1}=\left[\begin{array}{ll}
\mathbf{0} & \boldsymbol{J}_{s 2}
\end{array}\right]=\left[\begin{array}{cc}
0 & -0.3 / \sqrt{2} \\
0 & 0.3 / \sqrt{2} \\
0 & 0
\end{array}\right] .
$$

From Eq.(11), the RSP matrix and the reflection force can be calculated as:

$$
\begin{gathered}
\boldsymbol{R}_{E 1}=-K_{E}\left(\boldsymbol{I}-\boldsymbol{J}_{s 1 \ominus} \boldsymbol{J}_{s 1 \ominus}^{\#}\right) \boldsymbol{J}_{s} \boldsymbol{J}_{s}^{\#}=-\frac{K_{E}}{2}\left[\begin{array}{lll}
1 & 1 & 0 \\
1 & 1 & 0 \\
0 & 0 & 0
\end{array}\right], \\
\boldsymbol{F}_{R E 1}=\boldsymbol{R}_{E 1} \boldsymbol{e}_{s}=-\frac{K_{E}}{2}\left(e_{s x}+e_{s y}\right)\left[\begin{array}{lll}
1 & 1 & 0
\end{array}\right]^{T} .
\end{gathered}
$$

When the second joint is constrained, in the same manner,

$$
\boldsymbol{J}_{s \ominus 2}=\left[\begin{array}{cc}
-0.3 / \sqrt{2} & 0 \\
0.2+0.3 / \sqrt{2} & 0 \\
0 & 0
\end{array}\right] .
$$

The RSP matrix and the reflection force can be calculated as:

$$
\begin{aligned}
\boldsymbol{R}_{E 2}= & -K_{E}\left(\boldsymbol{I}-\boldsymbol{J}_{s 2 \ominus} \boldsymbol{J}_{s 2 \ominus}^{\#}\right) \boldsymbol{J}_{s} \boldsymbol{J}_{s}^{\#} \\
= & -K_{E}\left[\begin{array}{ccc}
0.791 & 0.407 & 0 \\
0.407 & 0.209 & 0 \\
0 & 0 & 0
\end{array}\right], \\
\boldsymbol{F}_{R E 2}= & \boldsymbol{R}_{E 2} \boldsymbol{e}_{s} \\
= & -K_{E}\left(1.92 e_{s x}+0.99 e_{s y}\right)\left[\begin{array}{c}
0.2+0.3 / \sqrt{2} \\
0.3 / \sqrt{2} \\
0
\end{array}\right] .
\end{aligned}
$$

Fig.3 illustrates the IMS and the IRS $S_{E}$ at the given pose in the 2-dimensional planar space. $\mathrm{IMS}_{E 1}$ and $\mathrm{IRS}_{E 1}$ are the IMS and IRS when the first joint is constrained. IMS $\mathrm{I}_{E 2}$ and IRS $_{E 2}$ are the IMS and IRS when the second joint is constrained. From Eq.(19) and Eq.(22), $\boldsymbol{F}_{R E 1}$ and $\boldsymbol{F}_{R E 2}$ reflect the forces in the direction of $\mathrm{IRS}_{E 1}$ and $\mathrm{IRS}_{E 2}$ respectively. Thus, the RSP method gives the accurate IRS.

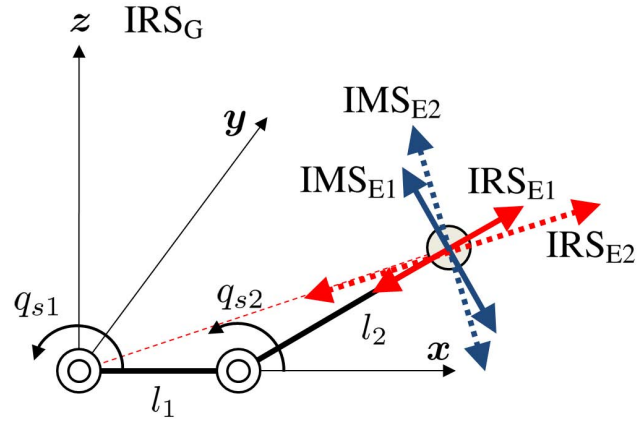

Fig. 3. $\operatorname{IRS}_{G}, \operatorname{IMS}_{E i}, \operatorname{IRS}_{E i}(i=1,2):$ IMS and IRS when the $i$ th joint of a 2 degrees-of-freedom manipulator is constrained

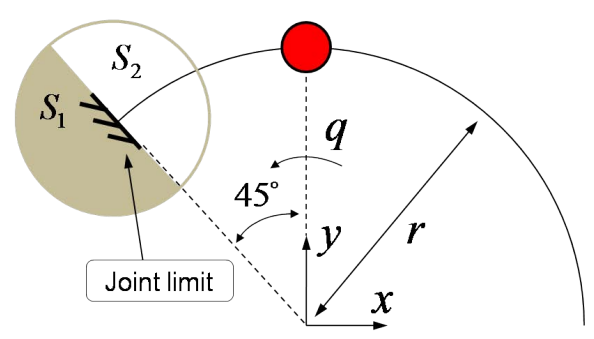

Fig. 4. A simple version of BiTESS-II: 1 degrees-of-freedom manipulator in 2 dimensional space. $S_{1}$ is not achievable space due to the joint limit. $S_{2}$ is a free space.

\section{B. Example 2: 1 degrees-of-freedom manipulator in 3 di- mensional space}

This example uses a simplified version of the spine bone fusion surgical manipulator, BiTESS-II [16], as shown in Fig.4 (KDS-RMASTER in Table I). The Jacobian of the manipulator is described as:

$$
\boldsymbol{J}_{s}=\left[\begin{array}{c}
-r \cos (q) \\
-r \sin (q) \\
0
\end{array}\right] .
$$

When $r=\sqrt{2}$ and $q=\pi / 4$, i.e, $x=-1$ and $y=-1, \boldsymbol{R}_{G}$ and $\boldsymbol{R}_{E}$ can be calculated from Eq.(9) and Eq.(11) as:

$$
\begin{aligned}
& \boldsymbol{R}_{G}=-K_{G}\left[\begin{array}{ccc}
0.5 & -0.5 & 0 \\
-0.5 & 0.5 & 0 \\
0 & 0 & 1
\end{array}\right] \\
& \boldsymbol{R}_{E}=-K_{E}\left[\begin{array}{ccc}
0.5 & 0.5 & 0 \\
0.5 & 0.5 & 0 \\
0 & 0 & 0
\end{array}\right] .
\end{aligned}
$$

Assume that the pose error is $\left[e_{s x}, e_{s y}, e_{s z}\right]^{T}$. If the pose error is in the half space, $S_{1}$, corresponding to the joint limit, then, the motion is constrained, resulting in a joint error. Therefore, when the desired command is in $S_{1}$ and the joint angle error is larger than $\varepsilon, \boldsymbol{F}_{R G}$ and $\boldsymbol{F}_{R E}$ can be calculated

\footnotetext{
${ }^{4}$ The subscriptions 1 and 2 are added to $\boldsymbol{J}_{s \ominus}, \boldsymbol{R}_{E}$, and $\boldsymbol{F}_{R E}$ for each condition.
} 


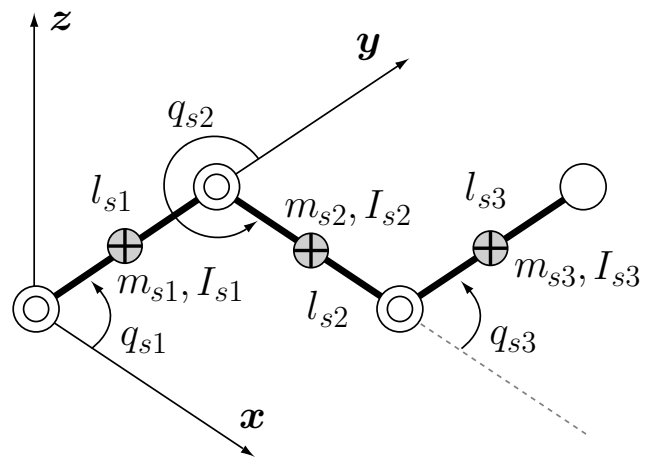

Fig. 5. A planar 3 degrees-of-freedom manipulator in 3-dimensional cartesian space.

from Eq.(24) and Eq.(25) as:

$$
\begin{aligned}
& \boldsymbol{F}_{R G}=\boldsymbol{R}_{G} \cdot\left[e_{s x}, e_{s y}, e_{s z}\right]^{T}=0.5 K_{G}\left[\begin{array}{c}
\left(e_{s x}-e_{s y}\right) \\
\left.-\left(e_{s x}-e_{s y}\right)\right], \\
e_{s z}
\end{array}\right], \\
& \boldsymbol{F}_{R E}=\boldsymbol{R}_{E} \cdot\left[e_{s x}, e_{s y}, e_{s z}\right]^{T}=0.5 K_{E}\left[\begin{array}{c}
\left(e_{s x}+e_{s y}\right) \\
\left(e_{s x}+e_{s y}\right) \\
0
\end{array}\right] .
\end{aligned}
$$

From Eq.(26), $\boldsymbol{F}_{R G}$ constrains the $z$-axis motion of the slave robot and the normal direction of the achievable path in $x-y$ plane.

When the desired command is in $S_{2}$, where the manipulator is within the joint limit, $\boldsymbol{F}_{R E}=0$, and there is no joint angle error. In Eq.(27), if the desired command is in $S_{1}$, the motion is constrained and the reflection force direction is always normal to the constraint space.

\section{Example 3: Three degrees-of-freedom manipulator in 3 dimensional space}

Consider a three degrees-of-freedom planar manipulator in 3-dimensional cartesian space (KDS-RSLAVE in Table I) as shown in Fig.5. When $l_{1}=l_{2}=l_{3}=1, q_{1}=\pi / 2$, $q_{2}=-\pi / 2$, and $q_{3}=2 \pi / 3$, the Jacobian is represented as:

$\boldsymbol{J}_{s}=\left[\begin{array}{lll}\boldsymbol{J}_{s 1} & \boldsymbol{J}_{s 2} & \boldsymbol{J}_{s 3}\end{array}\right]=\left[\begin{array}{ccc}-1-\sqrt{3} / 2 & -\sqrt{3} / 2 & -\sqrt{3} / 2 \\ 1 / 2 & 1 / 2 & -1 / 2 \\ 0 & 0 & 0\end{array}\right]$

In this example, we assume that $e_{s q 1}>\varepsilon, e_{s q 2}>\varepsilon$, and $e_{s q 3}<\varepsilon$ when the joint angle error is represented as $e_{s q}=$ $\left[e_{s q 1}, e_{s q 2}, e_{s q 3}\right]^{T}$. Thus, the first and the second joint are constrained simultaneously. Then,

$$
\boldsymbol{J}_{s \ominus}=\left[\begin{array}{lll}
\mathbf{0} & \mathbf{0} & \boldsymbol{J}_{s 3}
\end{array}\right]=\left[\begin{array}{ccc}
0 & 0 & -\sqrt{3} / 2 \\
0 & 0 & -1 / 2 \\
0 & 0 & 0
\end{array}\right]
$$

$\boldsymbol{R}_{E}$ can be calculated from Eq.(9) and Eq.(11) as:

$$
\begin{aligned}
& \boldsymbol{R}_{G}=K_{G}\left[\begin{array}{lll}
0 & 0 & 0 \\
0 & 0 & 0 \\
0 & 0 & 1
\end{array}\right] \\
& \boldsymbol{R}_{E}=K_{E}\left[\begin{array}{ccc}
1 / 4 & -\sqrt{3} / 4 & 0 \\
-\sqrt{3} / 4 & 3 / 4 & 0 \\
0 & 0 & 0
\end{array}\right] .
\end{aligned}
$$

Assume that the pose error is $\boldsymbol{e}_{s x}=\left[e_{s x}, e_{s y}, e_{s z}\right]^{T}$. Then, $F_{R G}$ and $F_{R E}$ can be calculated from Eq.(24) and Eq.(25) as:

$$
\begin{aligned}
& \boldsymbol{F}_{R G}=\boldsymbol{R}_{G} \boldsymbol{e}_{s}=K_{G}\left[0,0, e_{s z}\right]^{T}, \\
& \boldsymbol{F}_{R E}=\boldsymbol{R}_{E} \boldsymbol{e}_{s}=K_{E}\left(\sqrt{3} e_{s} y-e_{s x}\right) / 4\left[\begin{array}{c}
-1 \\
\sqrt{3} \\
0
\end{array}\right] .
\end{aligned}
$$

From Eq.(32), the motion in $z$-direction is constrained by $\boldsymbol{F}_{R G}$. From Eq.(33), the motion in the tangential direction of the third link, $q_{s 1}+q_{s 2}+q_{s 3}=2 \pi / 3$, is constrained.

\section{Discussion AND CONCLUding REMARKS}

The Restriction Space Projection method is simple and effective solution to provide the accurate direction of Instantaneous Restriction Space for position sensor based force reflection of any types of kinematically dissimilar multi degrees-of-freedom bilateral teleoperation systems. It operates robustly in unstructured environments where the slave robot can collide with unexpected obstacles at any point on body of the slaver robot not just the tip point of the endeffector, as typically assumed by bilateral teleoperation force reflection algorithm.

The RSP method guarantees the accurate direction of the restriction space but it cannot calculate the amplitude of the interaction force at the slave side. The amplitude needs to be adjusted by multiplying the projected position error by a scalar force gain, as it is typically done in position error based force feedback algorithm. If the force sensors placed on the robot are able to detect every possible collision including unexpected link collision, it promises better results than position error force feedback method. As such, the proposed RSP method is developed to enhance the conventional position sensor based force feedback, not to replace the force sensor based force feedback.

The application of the RSP method is limited to the restriction space generated by relatively high impedance obstacles that constraint the motion of the slaver robot. In other words, the RSP does not work if the motion of the slave robot is not constrained by obstacles (e.g, a powerful slave robot that can pushes obstacles without any disturbance or a slave robot with non back-drivable joints that is not affected by exogenous active constraints). It is a trade-off between the sensitivity of the force amplitude estimation and the cost of the force sensors. 


\section{REFERENCES}

[1] K. Kim, W. K. Chung, and M. C. Cavusoglu, "Description of Instantaneous Restriction Space for Multi-DOFs Bilateral Teleoperation Systems Using Position Sensors in Unstructured Environments ," IEEE Transactions on Robotics, vol. 25, no. 5, pp. 1150-1158, 2009.

[2] K. Kim and W. K. Chung, "Accurate force reflection for kinematically dissimilar bilateral teleoperation systems using instantaneous restriction space," in Proceedings of the IEEE Interational Conference on Robotics and Automation, Orlando, USA, 2006, pp. 3257-3262.

[3] K. Kim, W. Chung, and S. Nam, "Accurate force reflection method for a multi-dof haptic interface using instantaneous restriction space without a force sensor in an unstructured environment," Advanced Robotics, vol. 21, no. 1, pp. 87-104, 2007.

[4] A. Liegeois, "Automatic supervisory control of the configuration and behavior of multibody mechanisms," vol. 7, no. 12, pp. 868-871, 1977.

[5] Y. Nakamura, Advanced Robotics: Redundancy and Optimization. Addison-Wesley, 1991.

[6] B. Hannaford, "A design framework for teleoperators with kinesthetic feedback," IEEE Transactions on Robotics and Automation, vol. 5, no. 4, pp. 426-434, 1989.

[7] R. J. Anderson and M. W. Spong, "Bilateral control of teleoperators with time delay," IEEE Transactions on Automatic Control, vol. 34, no. 5, pp. 494-501, 1989.

[8] G. Niemeyer and J. Slotine, "Stable adaptive teleoperation," IEEE Journal of Oceanic Engineering, vol. 16, pp. 152-162, 1991.

[9] D. A. Lawrence, "Stability and transparency in bilateral teleoperation," IEEE Transactions on Robotics and Automation, vol. 9, no. 5, pp. 624637, 1993.

[10] Y. Yokokohji and T. Yoshikawa, "Bilateral control of master-slave manipulators for ideal kinesthetic coupligng-formulation and experiment," IEEE Transactions on Robotics and Automation, vol. 10, no. 5, pp. 605-620, 1994.

[11] J. E. Colgate and J. M. Brown, "Factors affecting the z-width of a haptic display," in Proceedings of the IEEE Interational Conference on Robotics and Automation, May 1994, pp. 3205-3210.

[12] R. J. Adams and B. Hannaford, "Stable haptic interaction with virtual environment," IEEE Transactions on Robotics and Automation, vol. 15, no. 3, pp. 465-474, 1999.

[13] B. Hannaford and J. H. Ryu, "Time-domain passivity control of haptic interfaces," IEEE Transactions on Robotics and Automation, vol. 18, no. 1, pp. 1-10, 2002.

[14] K. H. Zaad and S. E. Salcudean, "Transparency in time-delayed systems and the effect of local force feedback for transparent teleoperation," IEEE Transaction on Robotics and Automation, vol. 18, no. 1, pp. 109-114, 2002.

[15] M. C. Cavusoglu, A. Sherman, and F. Tendick, "Design of bilateral teleoperation controllers for haptic exploration and tele manipulation of soft environment," IEEE Transactions on Robotics and Automation, vol. 18 , no. 4, pp. 641-647, 2002.

[16] K. Kim, J. Lee, W. Chung, S. Choi, Y. Kim, and I. Suh, "A Noble Bilateral Teleoperation System for Human Guided Spinal Fusion," Robotics and Automation, 2007 IEEE International Conference on, pp. 940-946, 2007. 